\title{
Circulating microRNAs as potential new biomarkers for prostate cancer
}

\author{
A Sita-Lumsden ${ }^{1}$, D A Dart ${ }^{1}$, J Waxman ${ }^{1}$ and C L Bevan ${ }^{\star}, 1$ \\ ${ }^{1}$ Androgen Signalling Laboratory, Department of Surgery and Cancer, Imperial College London, London W12 ONN, UK
}

Since they were first described in the 1990s, circulating microRNAs (miRNAs) have provided an active and rapidly evolving area of current research that has the potential to transform cancer diagnostics and therapeutics. In particular, miRNAs could provide potential new biomarkers for prostate cancer, the most common cause of cancer in UK men. Current diagnostic tests for prostate cancer have low specificity and poor sensitivity. Further, although many prostate cancers are so slow growing as not to pose a major risk to health, there is currently no test to distinguish between these and cancers that will become aggressive and life threatening. Circulating miRNAs are highly stable and are both detectable and quantifiable in a range of accessible bio fluids, thus have the potential to be useful diagnostic, prognostic and predictive biomarkers. This review aims to summarise the current understanding of circulating miRNAs in prostate cancer patients and their potential role as biomarkers.

\section{PROSTATE CANCER NEEDS NEW BIOMARKERS}

Prostate cancer is the most commonly diagnosed male cancer in the Western world, with more than 40000 cases in the United Kingdom diagnosed annually, and its management would benefit greatly from new biomarkers for diagnosis, prognosis and treatment response. Prostate-specific antigen (PSA) detection in serum is the current gold standard biomarker for diagnosis and response to treatment, but it has well-known limitations as a biomarker. In terms of a diagnostic marker, elevated serum PSA is not specific to malignant prostate disease and $>50 \%$ of men undergoing biopsy following a raised PSA result are diagnosed with prostate cancer, an unacceptably high false-positive rate given the risks of biopsy. PSA testing also has a false-negative rate of approximately 15\% (Schroder and Roobol, 2009). PSA has little value as a prognostic biomarker as screening detects indolent tumours as well as those that are or will become life threatening. The majority of men with prostate cancer die of other causes, and the costs and morbidity associated with over-treating these indolent prostate tumours amount to a significant public health problem. In addition, up to a quarter of men treated with curative intent for localised prostate cancer experience relapse within 5 years (Greene et al, 2004). This lack of sensitivity and specificity of PSA testing has contributed to controversy over the value of early detection. Two large multi-national randomised control trials, the European Randomised Study of Screening in Prostate Cancer and the Prostate, Lung, Colorectal and Ovarian Screening Trial provided conflicting results as to whether PSA screening for prostate cancer reduces mortality. In addition, it is unclear as to whether PSA screening may actually contribute to over diagnosis and overtreatment of prostate cancer (Schroder and Roobol, 2009).

There are a variety of tools to improve the clinical usefulness of PSA by incorporating it with other markers into an algorithm. One such scoring system- 'the Cancer of the Prostate Risk Assessment' (CAPRA) score is used to aid clinical decisions regarding treatments at the time of diagnosis, as a surrogate prognostic biomarker (Cooperberg et al, 2005). This algorithm is based on patient's age, histological Gleason tumour grade, staging of the tumour, percentage of biopsy cores positive for cancer at diagnosis and the initial PSA level. The CAPRA score has been externally and independently verified and is routinely used to guide treatment choices, but this test algorithm also lacks specificity and sensitivity (May et al, 2007).

Therapeutic decision-making in hormone refractory prostate cancer is a challenge due to frequent and inconsistent changes in serum PSA, patient symptoms and radiographic findings. A decision to discontinue ineffective agents early decreases morbidity from drug toxicity, reduces costs and allows the patient to try an alternative therapy. Further, validation of a biomarker as a robust surrogate end point to replace overall survival would also potentially speed up the drug development pathway. To date, circulating tumour cells have been shown to be the strongest

*Correspondence: Dr CL Bevan; E-mail: charlotte.bevan@imperial.ac.uk

Received 29 October 2012; revised 19 March 2013; accepted 28 March 2013;

published online 30 April 2013

(C) 2013 Cancer Research UK. All rights reserved 0007-0920/13
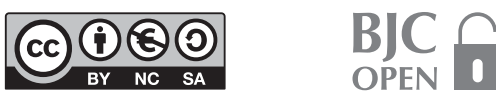
independent predictor of overall survival, more predictive than post-therapy PSA (de Bono et al, 2008). Given the recent findings discussed below, in the near future micro-ribonucleic acids (miRNAs) could have a role alongside circulating tumour cells and PSA in prediction of response to therapy.

\section{MIRNAS AS POTENTIAL BIOMARKERS}

MiRNAs are short RNA molecules, on average 22 nucleotides long. First discovered in 1993, they function as post-transcriptional regulators that bind to complementary sequences on target mRNA usually in the $3^{\prime} U T R$. This usually results in translational repression or mRNA degradation resulting in reduced levels of encoded protein; however, they can also induce gene expression by binding to complementary promoter sequences thus upregulating translation (reviewed in Nicoloso et al, 2009). The system is complex; genes can be targeted by multiple miRNAs and each miRNA is able to target hundreds of transcripts either directly or indirectly. To date, over 1900 mature human miRNAs have been identified (Kozomara and Griffiths-Jones, 2011). The importance of miRNAs in gene regulation is highlighted by the observation that loss of Dicer, an RNase required for miRNA biogenesis, results in embryonic lethality in mice (Bernstein et al, 2003). There is a growing and an increasingly convincing body of evidence that miRNAs are frequently overexpressed or downregulated in the malignant process and that miRNAs may therefore function as either oncomiRs, encouraging tumour growth, or tumour suppressors, repressing tumour growth (reviewed in Heneghan et al, 2010).

MiRNAs may exert their aberrant activity either via quantitative effects, such as a change in expression level or by qualitative effects that occur when there are losses or gains to a recognition sequence either within the miRNA or its target. The function of a given miRNA is largely determined by the relative availability of the target mRNAs, as such individual miRNAs can have disparate effects in differing tissues, specifically cancers of different cellular origin. An example of this is miRNA-125b, which has been shown to be an oncomiR in prostate cancer and to act as a tumour suppressor in ovarian and breast cancer (reviewed in Cortez et al, 2011).

Some of the innate properties of miRNAs make them highly attractive as potential biomarkers. MiRNAs can be readily detected in small volume samples using specific and sensitive quantitative real-time PCR (qRT-PCR); they have been isolated from most body fluids, including serum, plasma, urine, saliva, breast milk, tears and semen and are known to circulate in a highly stable, cellfree form (Cortez et al, 2011). They are highly conserved between species, allowing the use of animal models of disease for preclinical studies. Furthermore, tumour cells have been shown to release miRNAs into the circulation (Taylor and Gercel-Taylor, 2008) and profiles of miRNAs are altered in the plasma and/or serum of patients with cancer (Taylor and Gercel-Taylor, 2008).

\section{ROLE AND TRANSPORTATION OF CIRCULATING MIRNAS}

Although it is clear that miRNAs function as a mechanism for post-transcriptional regulation, it has not been conclusively proven whether their presence in circulation is simply a by-product of cell degradation or whether are they actively secreted into the body fluids to mediate intercellular gene regulation. A body of evidence supports the hypothesis that miRNAs can be actively and selectively secreted; for example, miR-1246 and miR-451 have been found to be released by the breast cancer cell line MCF-7 but not by the non-malignant mammary epithelial breast cell line (Pigati et al, 2010).

Also in support of active secretion is the appropriate packaging of miRNAs to facilitate circulation and protect them from degradation in body fluids. MiRNAs in serum are resistant to circulating ribonucleases and severe physicochemical conditions, such as extended storage, freeze-thawing and extreme $\mathrm{pH}$ (Chen et al, 2008; Mitchell et al, 2008). There are three known methods by which miRNAs are packaged: in lipid vesicles, such as exosomes and apoptotic bodies; bound by RNA-binding proteins, such as nucleophosmin 1 and Argonaute 2; and associated with highdensity lipoprotein. Such packaging of miRNAs has a wider relevance as it facilitates the transfer of miRNAs between individuals as exemplified by the example of immune-related miRNAs in breast milk in the first 6 months of lactation, corresponding with the passive immunity gained from colostrum in the first 6 months. The implication of these findings for treatment is that miRNAs, packaged appropriately, could be given orally and not digested (Iguchi et al, 2010). These structurally diverse extracellular miRNA-containing entities may also allow target enrichment and therefore improve the utility of miRNAs as biomarkers. For example, extraction of tissue-specific exosomes using protein surface markers could potentially enrich for a cancerspecific miRNA population, as has been shown in ovarian cancer (Taylor and Gercel-Taylor, 2008).

\section{METHODOLOGICAL CHALLENGES IN MEASURING} CIRCULATING MIRNAS

A prerequisite to developing circulating miRNA-based diagnostics is the ability to quantify miRNAs from plasma and serum with sufficient sensitivity and precision. The accurate measurement of these miRNAs has been associated with many challenges, including those related to pre-analytic variation such as specimen collection, factors influencing RNA extraction efficiency and technical issues involved in successful qRT-PCR and data analysis and normalisation (Kroh et al, 2010).

Major reasons for the variation and lack of consistency in circulating miRNA data to date are likely the many potentially confounding variables in methodology of extraction and quantification of miRNAs and data analysis. For instance, the use of blood plasma or serum: there is a higher risk of cellular contamination when preparing plasma as the supernatant is pipetted away from the cellular pellet. The type of anticoagulant used: EDTA and citrate are acceptable, but heparin impedes the qRT-PCR step. There is also considerable inter-sample variability in both the protein and lipid content of each individual's serum and plasma, which could affect the efficiency of RNA extraction and potentially introduce potential inhibitors to qRT-PCR. Other variables of note that need to be standardised are the duration of time between blood collection and processing. The actual isolation of miRNAs from serum and plasma is relatively straightforward, using a guanidinium-phenol extraction followed by either precipitation of the miRNA-containing aqueous phase or column-based purification (Kroh et al, 2010).

The main issues that remain unresolved in the measuring of circulating miRNAs are normalisation, amplification and contamination. Quantitating the amount of total miRNA in body fluid specimens is not possible because of the extremely low concentrations. In addition, there is no consensus on suitable small RNA reference genes that could be used as internal controls for biological variability; current protocols call for samples to be processed from identical input volumes, then corrected for technical variability using spiked-in synthetic non-human (Caenorhabditis elegans) miRNA as a normalising control (Mitchell et al, 2008; Kroh et al, 2010). Use of 'invariant' miRNAs 
as endogenous controls has been proposed by some investigators, but biological variability may preclude this approach and no consensus on which has been reached to date. Another area requiring standardisation is the use of pre-amplification reagents to increase the sensitivity of the qRT-PCR, used in some studies but not in others. Lastly, quantification of valid miRNAs in the serum can be grossly altered by contamination by miRNAs leaked from cellular blood components, either through haemolysis during sampling or processing or by carry-over of whole cells in the serum/plasma. Given all the uncertainties surrounding miRNA extraction and analysis, it is clear why miRNA analysis is yet to be implemented in the clinic: until there is standardisation in phlebotomy protocol, sample processing, miRNA extraction methodology and reliable quantitation, the translatability of these studies will be limited (Kroh et al, 2010). However, the studies published to date do point towards miRNAs having a role in the future of prostate cancer biomarkers and will form a solid base upon which to design future studies based on consensus protocols.

MIRNAS AS DIAGNOSTIC BIOMARKERS IN PROSTATE CANCER

The first report of miRNAs as potential diagnostic markers was in 2008. Mitchell et al (2008) probed a panel of miRNAs in the serum of healthy men and those with advanced prostate cancer and found that miR-141 was highly elevated in the cancer samples. Moreover, miR-141 was found to correlate significantly with serum PSA levels and could detect individuals with advanced prostate cancer with $60 \%$ sensitivity and $100 \%$ specificity. The fact that Mitchell et al (2008) compared healthy controls with advanced (metastatic) cancer could explain the difference with a later study by Yaman Agaoglu et al (2011) who found that miR-141 was not higher in 26 patients with localised disease compared with 20 healthy individuals. They did, however, find that miR-21 and -221 were significantly higher in the early-stage disease compared with controls. Later, Lodes et al (2009) used a custom microarray to profile miRNAs in serum from patients with various cancer types. Although they identified 15 miRNAs elevated in the prostate cancer patients, the profile was unable to discriminate between prostate cancer patients and those patients with other malignancies, such as breast or colorectal cancer.

Recently high-throughput multiplexed qRT-PCR has been used to identify miRNAs significantly altered in the serum of patients with prostate cancer. Moltzahn et al (2011) compared 36 earlystage prostate cancer patients immediately before prostatectomy with 12 healthy men. Receiver operated curves generated for the individual miRNAs showed that some possessed significant diagnostic capability. Three-miR-93, miR-106a and miR-24showed consistently low levels in the healthy individuals $v s$ high levels in the cancer groups, respectively. Bryant et al (2012) found 12 miRNAs were altered in the circulation of 78 men with prostate cancer compared with 28 healthy men-miR-107 had the greatest fold change. Chen et al (2012) defined a five-miRNA panel (downregulation of let-7e, let-7c and miR-30c, upregulation of miR-622 and miR-1285) with diagnostic value, able to differentiate between 80 patients with prostate cancer and 44 patients with benign prostatic hyperplasia (area under the curve $=0.924$ ).

A 2012 study by Selth et al (2012), which showed miR-141, miR-298, miR-346 and miR-375 to be consistently elevated in patients with advanced prostate cancer over healthy individuals, is notable in that they identified these candidate miRs first in the Transgenic Adenocarcinoma of Mouse Prostate (TRAMP) mouse model of prostate cancer. The authors argue that this, also in light of the process of cancer progression in this model in many respects mirroring human disease and the extensive functional homology between mouse and murine miRs, demonstrates the potential for mouse models to be used in identification of circulating miR biomarkers, although they also report six other miRs to be upregulated in TRAMP mice but not the human cancer patients (Selth et al, 2012).

Mahn et al (2011) found that miR-26a, miR-195 and let-7i were increased in the serum of men with localised prostate cancer when compared with men with benign prostatic hyperplasia (area under the curve $=0.758$ ); however, when compared with healthy individuals none of the candidate miRNAs were significantly altered. This study highlights the importance of age-matched controls, as these miRNAs are likely to be altered with ageing and hormonal state. This study also supports the tumoural association of these miRNAs, as the levels of miRNA in prostate cancer tissue were highly correlated with levels in the serum. Furthermore, levels decreased significantly in the serum following surgery. This is of particular relevance in prostate cancer, as some miRNAs (e.g., miR-141 and miR-27a) have been shown to be under androgen control; therefore they are likely to be expressed at different levels depending on the treatment undergone by the patient (Waltering et al, 2011; Fletcher et al, 2012).

MIRNAS AS PROGNOSTIC BIOMARKERS IN PROSTATE CANCER

As already discussed, current attempts to distinguish the subgroup of prostate cancer patients who will relapse post 'curative treatment' with metastatic disease is based on an evaluation of a collection of markers, including PSA, Gleason score and histological score. Brase et al (2011) set out to establish markers of micrometastasis in prostate cancer by comparing serum from men with primary prostate cancer with those with metastatic prostate cancer. They found 69 miRNAs elevated in men with metastatic disease; a subset of these was then measured in the men with localised prostate cancer. Three-miR-141, miR-200b and miR-375-were found to be elevated with increasing tumour stage and Gleason score. A similar study conducted by Bryant et al (2012) compared plasma from patients with localised prostate cancer with patients with metastatic prostate cancer, and found 16 miRNAs, including miR-141, miR-200b and miR-375, to be differentially expressed, and miR-141, miR-21 and miR-221 were also significantly higher in serum of advanced $v$ localised prostate cancer patients in the previously mentioned study by Yaman Agaoglu et al (2011). Moltzahn et al (2011) compared serum collected before treatment from 29 patients with differing CAPRA scores. MiR-24 levels were found to decrease with increasing CAPRA score, whereas miR-106a, miR-451 and miR-93 were increased in association with the CAPRA score. In another study of 82 prostate cancer patients, miR-20a and miR-21 were significantly increased in patients with a high-risk CAPRA score (Shen et al, 2012).

These prognostic biomarker studies highlight miR-141, miR$200 \mathrm{~b}$ and miR-375 as significant disease correlates, which could potentially be used in a test at the time of diagnosis to identify those patients with previously undetectable micrometastases. Future studies with longitudinal follow-up would allow us to compare initial serum miRNA levels at the time of localised disease between the cohort that go on to develop metastasis and those that do not.

\section{MIRNAS AS PREDICTIVE BIOMARKERS IN PROSTATE} CANCER

Predicting a patient's response to treatment would allow tailoring of appropriate regimens and a reduction in treatment-induced morbidity. Zhang et al (2011) looked specifically at levels of 
miR-21 in patients with localised prostate cancer, androgendependent prostate cancer (ADPC), castrate-resistant prostate cancer (CRPC) and benign prostatic hyperplasia. Levels of this miRNA were found to be significantly higher in CRPC and ADPC in those patients with a PSA $>4 \mathrm{ng} \mathrm{ml}^{-1}$. Most interestingly, four CRPC patients who were resistant to docetaxel chemotherapy had significantly higher miR-21 levels than the six patients with responsive disease. Potentially, miRNAs or miRNA profiles could be used to predict response to a given therapy and further could give clues as to mechanisms of resistance.

The utility of miR-141 as a biomarker of treatment response was assessed by Gonzales et al (2011) who directly compared it with PSA and found both miR-141 and PSA had a sensitivity of 78.9\%, whereas the specificity of miR-141 was $68.8 \%$ compared with $87.4 \%$ for PSA in predicting clinical progression in 21 patients with CRPC or ADPC receiving a range of therapies. This longitudinal behaviour and predictive ability was most closely related to, but still distinct from, PSA with a significant positive correlation $(R=0.77, \quad P<0.001)$. Although miR-141 does not add any additional prognostic information, this study in a small cohort of men highlights the potential of circulating miRNAs for evaluating treatment efficiency.

\section{MIRNAS AS TREATMENT TARGETS}

The elucidation of those miRNAs involved in the pathogenesis of prostate cancer could potentially help to identify targets for future therapies. Some of the miRNAs discussed are elevated both in circulation and in prostate tumour tissue, notably miR-375 and miR-141. Szczyrba et al (2011) showed that miR-375 targets SEC23a, a putative tumour suppressor, while MiR-141 is a member of the miR-200 family, which targets the ZEB family of transcription factors, which function to repress the epithelial-tomesenchymal transition, a key step in cancer metastasis (Gonzales et al, 2011). It has also been shown that miR-27a targets the tumour-suppressor prohibitin in both prostate cancer and gastric cancer, and reducing its levels inhibited the growth of prostate cancer cells (Liu et al, 2009; Fletcher et al, 2012). A complication is that each miRNA potentially targets many different mRNAs, thus manipulating a given miRNA could alter the protein products in several signal transduction pathways resulting in potential offtarget effects. However, this could also constitute an advantagefor instance, miR-27a targets several tumour-suppressor genes (e.g., Prohibitin, Wee1, ZBTB10, FOXO1, reviewed in Fletcher

Table 1. Synopsis of results of published studies investigating the potential of circulating miRNAs as biomarkers of prostate cancer

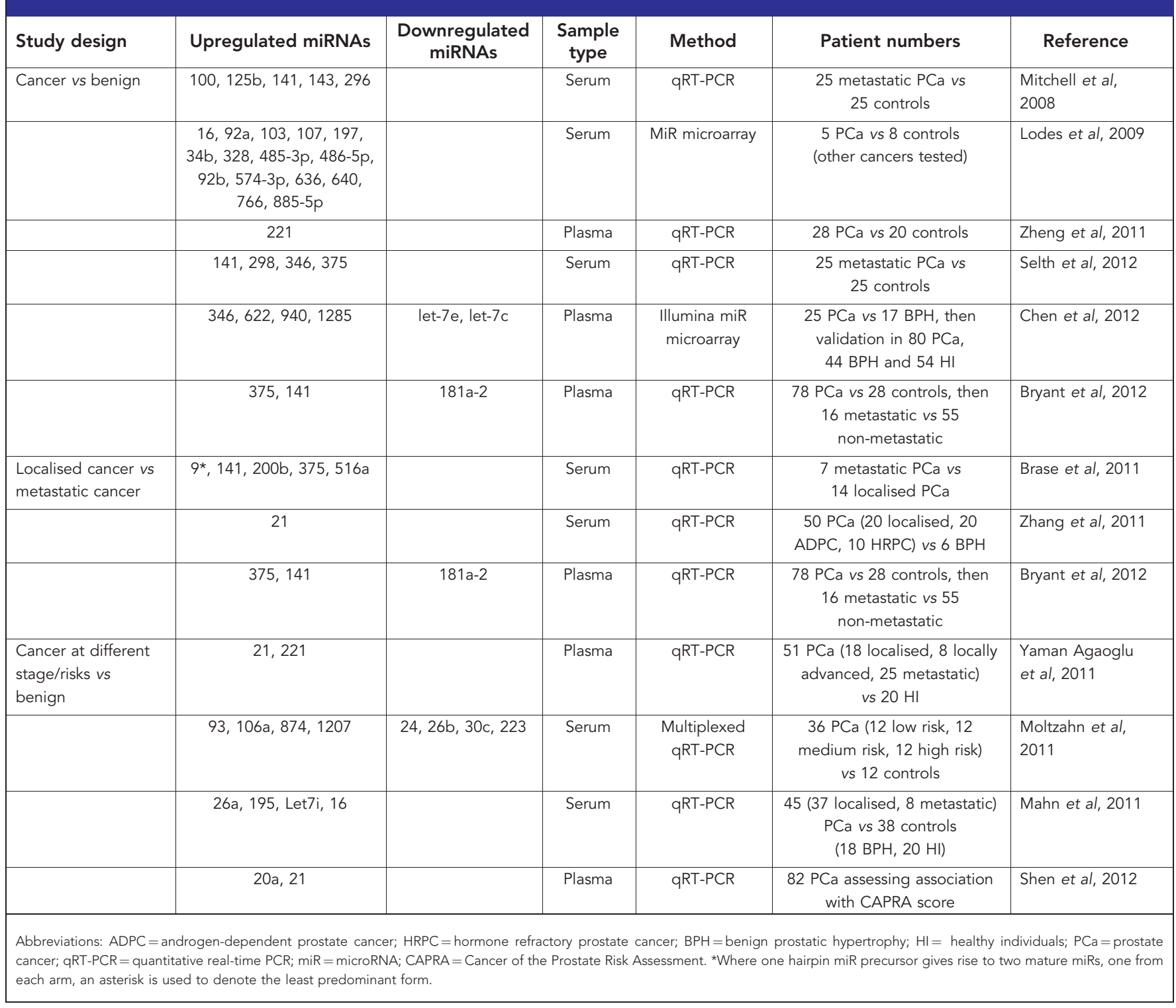


et al. 2012) and thus inhibiting this miR could inhibit tumour growth via several pathways. Further exploration of the functions and targets of such miRNAs will reveal in time the viability of miRNA targets as a therapeutic strategy.

\section{CONCLUSION}

There is considerable excitement surrounding the use of miRNAs in the biomarker field, which is well founded given their stability and relative ease of detection. MiRNAs with potential for use as diagnostic, prognostic, predictive biomarkers or indeed as a target for treatment are summarised in Table 1. However, currently their use is limited by conflicting data between studies due to lack of standardisation in methodology and the lack of suitable reference genes for normalisation. It is unlikely that any single miRNA will achieve the desired level of diagnostic or prognostic accuracy, as evidence is increasing that increased levels of particular miRNAs can be associated with several different types of tumour. We believe it will not be long, however, before these challenges are overcome and a miRNA-based diagnostic test is launched for prostate cancer, as has recently been done for pancreatic adenocarcinoma (Szafranska-Schwarzbach et al, 2011).

\section{ACKNOWLEDGEMENTS}

ASL was supported by Cancer Research UK (C42671/A12990) and AD by Prostate Cancer UK (PG10-25) during the writing of this minireview. We thank members of the Androgen Signalling Laboratory for advice and critical evaluation.

\section{REFERENCES}

Bernstein E, Kim SY, Carmell MA, Murchison EP, Alcorn H, Li MZ, Mills AA, Elledge SJ, Anderson KV, Hannon GJ (2003) Dicer is essential for mouse development. Nat Genet 35(3): 215-217.

Brase JC, Johannes M, Schlomm T, Falth M, Haese A, Steuber T, Beissbarth T, Kuner R, Sultmann H (2011) Circulating miRNAs are correlated with tumor progression in prostate cancer. Int J Cancer 128(3): 608-616.

Bryant RJ, Pawlowski T, Catto JW, Marsden G, Vessella RL, Rhees B, Kuslich C, Visakorpi T, Hamdy FC (2012) Changes in circulating microRNA levels associated with prostate cancer. Br J Cancer 106(4): 768-774.

Chen X, Ba Y, Ma L, Cai X, Yin Y, Wang K, Guo J, Zhang Y, Chen J, Guo X, Li Q, Li X, Wang W, Wang J, Jiang X, Xiang Y, Xu C, Zheng P, Zhang J, Li R, Zhang H, Shang X, Gong T, Ning G, Zen K, Zhang CY (2008) Characterization of microRNAs in serum: a novel class of biomarkers for diagnosis of cancer and other diseases. Cell Res 18(10): 997-1006.

Chen ZH, Zhang GL, Li HR, Luo JD, Li ZX, Chen GM, Yang J (2012) A panel of five circulating microRNAs as potential biomarkers for prostate cancer. Prostate 72(13): 1443-1452.

Cooperberg MR, Pasta DJ, Elkin EP, Litwin MS, Latini DM, Du Chane J, Carroll PR (2005) The University of California, San Francisco Cancer of the Prostate Risk Assessment score: a straightforward and reliable preoperative predictor of disease recurrence after radical prostatectomy. J Urol 173(6): 1938-1942.

Cortez MA, Bueso-Ramos C, Ferdin J, Lopez-Berestein G, Sood AK, Calin GA (2011) MicroRNAs in body fluids-the mix of hormones and biomarkers. Nat Rev Clin Oncol 8(8): 467-477.

de Bono JS, Scher HI, Montgomery RB, Parker C, Miller MC, Tissing H, Doyle GV, Terstappen LW, Pienta KJ, Raghavan D (2008) Circulating tumor cells predict survival benefit from treatment in metastatic castrationresistant prostate cancer. Clin Cancer Res 14(19): 6302-6309.

Fletcher CE, Dart DA, Sita-Lumsden A, Cheng H, Rennie PS, Bevan CL (2012) Androgen-regulated processing of the oncomir MiR-27a, which targets Prohibitin in prostate cancer. Human Mol Genet 21(14): 3112-3127.
Gonzales JC, Fink LM, Goodman Jr. OB, Symanowski JT, Vogelzang NJ, Ward DC (2011) Comparison of circulating MicroRNA 141 to circulating tumor cells, lactate dehydrogenase, and prostate-specific antigen for determining treatment response in patients with metastatic prostate cancer. Clin Genitourin Cancer 9(1): 39-45.

Greene KL, Meng MV, Elkin EP, Cooperberg MR, Pasta DJ, Kattan MW, Wallace K, Carroll PR (2004) Validation of the Kattan preoperative nomogram for prostate cancer recurrence using a community based cohort: results from cancer of the prostate strategic urological research endeavor (capsure). J Urol 171(6 Pt 1): 2255-2259.

Heneghan HM, Miller N, Kerin MJ (2010) MiRNAs as biomarkers and therapeutic targets in cancer. Curr Opin Pharmacol 10(5): 543-550.

Iguchi H, Kosaka N, Ochiya T (2010) Secretory microRNAs as a versatile communication tool. Commun Integr Biol 3(5): 478-481.

Kozomara A, Griffiths-Jones S (2011) miRBase: integrating microRNA annotation and deep-sequencing data. Nucleic Acids Res 39 (Database issue): D152-D157.

Kroh EM, Parkin RK, Mitchell PS, Tewari M (2010) Analysis of circulating microRNA biomarkers in plasma and serum using quantitative reverse transcription-PCR (qRT-PCR). Methods 50(4): 298-301.

Liu T, Tang H, Lang Y, Liu M, Li X (2009) MicroRNA-27a functions as an oncogene in gastric adenocarcinoma by targeting prohibitin. Cancer Lett 273(2): 233-242.

Lodes MJ, Caraballo M, Suciu D, Munro S, Kumar A, Anderson B (2009) Detection of cancer with serum miRNAs on an oligonucleotide microarray. PLoS One 4(7): e6229.

Mahn R, Heukamp LC, Rogenhofer S, von Ruecker A, Muller SC, Ellinger J (2011) Circulating microRNAs (miRNA) in serum of patients with prostate cancer. Urology 77(5): 1265, e9-e16.

May M, Knoll N, Siegsmund M, Fahlenkamp D, Vogler H, Hoschke B, Gralla O (2007) Validity of the CAPRA score to predict biochemical recurrence-free survival after radical prostatectomy. Results from a european multicenter survey of 1,296 patients. J Urol 178(5): 1957-1962, discussion 1962.

Mitchell PS, Parkin RK, Kroh EM, Fritz BR, Wyman SK, PogosovaAgadjanyan EL, Peterson A, Noteboom J, O'Briant KC, Allen A, Lin DW, Urban N, Drescher CW, Knudsen BS, Stirewalt DL, Gentleman R, Vessella RL, Nelson PS, Martin DB, Tewari M (2008) Circulating microRNAs as stable blood-based markers for cancer detection. Proc Natl Acad Sci USA 105(30): 10513-10518.

Moltzahn F, Olshen AB, Baehner L, Peek A, Fong L, Stoppler H, Simko J, Hilton JF, Carroll P, Blelloch R (2011) Microfluidic-based multiplex qRTPCR identifies diagnostic and prognostic microRNA signatures in the sera of prostate cancer patients. Cancer Res 71(2): 550-560.

Nicoloso MS, Spizzo R, Shimizu M, Rossi S, Calin GA (2009) MicroRNAsthe micro steering wheel of tumour metastases. Nat Rev Cancer 9(4): 293-302.

Pigati L, Yaddanapudi SC, Iyengar R, Kim DJ, Hearn SA, Danforth D, Hastings ML, Duelli DM (2010) Selective release of microRNA species from normal and malignant mammary epithelial cells. PLoS One 5(10): e13515.

Schroder FH, Roobol MJ (2009) Defining the optimal prostate-specific antigen threshold for the diagnosis of prostate cancer. Curr Opin Urol 19(3): $227-231$.

Selth LA, Townley S, Gillis JL, Ochnik AM, Murti K, Macfarlane RJ, Chi KN, Marshall VR, Tilley WD, Butler LM (2012) Discovery of circulating microRNAs associated with human prostate cancer using a mouse model of disease. Int J Cancer 131(3): 652-661.

Shen J, Hruby GW, McKiernan JM, Gurvich I, Lipsky MJ, Benson MC, Santella RM (2012) Dysregulation of circulating microRNAs and prediction of aggressive prostate cancer. Prostate 72(13): 1469-1477.

Szafranska-Schwarzbach AE, Adai AT, Lee LS, Conwell DL, Andruss BF (2011) Development of a miRNA-based diagnostic assay for pancreatic ductal adenocarcinoma. Expert Rev Mol Diagn 11(3): 249-257.

Szczyrba J, Nolte E, Wach S, Kremmer E, Stohr R, Hartmann A, Wieland W, Wullich B, Grasser FA (2011) Downregulation of Sec23A protein by miRNA-375 in prostate carcinoma. Mol Cancer Res 9(6): 791-800.

Taylor DD, Gercel-Taylor C (2008) MicroRNA signatures of tumor-derived exosomes as diagnostic biomarkers of ovarian cancer. Gynecol Oncol 110(1): 13-21. 
Waltering KK, Porkka KP, Jalava SE, Urbanucci A, Kohonen PJ, Latonen LM, Kallioniemi OP, Jenster G, Visakorpi T (2011) Androgen regulation of micro-RNAs in prostate cancer. Prostate 71(6): 604-614.

Yaman Agaoglu F, Kovancilar M, Dizdar Y, Darendeliler E, Holdenrieder S, Dalay N, Gezer U (2011) Investigation of miR-21, miR-141, and miR-221 in blood circulation of patients with prostate cancer. Tumour Biol 32(3): 583-588.

Zhang HL, Yang LF, Zhu Y, Yao XD, Zhang SL, Dai B, Zhu YP, Shen YJ, Shi GH, Ye DW (2011) Serum miRNA-21: elevated levels in patients with metastatic hormone-refractory prostate cancer and potential predictive factor for the efficacy of docetaxel-based chemotherapy. Prostate 71(3): $326-331$.

(c) (i) (2) This work is licensed under the Creative Commons (c) ${ }_{\text {BY }}$ NC sA Attribution-NonCommercial-Share Alike 3.0 Unported License. To view a copy of this license, visit http://creativecommons. org/licenses/by-nc-sa/3.0/ 